\title{
Supramolecular organic and inorganic photochemistry: Radical pair recombination in micelles, electron transfer on starburst dendrimers, and the use of DNA as a molecular wire
}

\author{
Nicholas J. Turro \\ Department of Chemistry, Columbia University, New York, NY 10027 (USA)
}

\begin{abstract}
Supramolecular chemistry is concerned with systems for which noncovalent interactions become significant or dominate in determining the chemistry of the guest/host systems. Supramolecular photochemistry is concerned with systems where non-covalent interactions become significant or dominate in determining the observed photochemistry. The supramolecular photochemistry of ketones adsorbed in micelles and of metal complexes adsorbed on starburst dendrimers and DNA is the subject of this report.
\end{abstract}

Introduction: From Molecular Photochemistry to Supramolecular Photochemistry.

Supramolecular chemistry has been defined as "chemistry beyond the molecule" 1 . Over the past two decades there has been an explosion in a field that can be aptly termed supramolecular photochemistry. $1 \mathrm{a}, \mathrm{b}$ Intense activity in the field has been driven by a number of factors, one of the most important of which is the exciting and stimulating intellectual basis of the supramolecular concepts which allow the analysis of complex aggregates of many molecules to be treated as a single intellectual unit, the supermolecule. We start with an overview of our structural approach to supramolecular chemistry and then show how it has been extended to energize and guide our research program in supramolecular photochemistry.

Supramolecular systems often possess many of the features of guest-host complexes. Thus, the familiar ideas of guest-host systems allow the use of the following metaphor to guide our thinking of supramolecular systems: supramolecular systems are related to molecules (through non-covalent intermolecular bonds) as molecules are related to atoms (through covalent intramolecular bonds): In this metaphor, the guest is often a small molecule of dimensions of several $\AA$ and the host is a large molecule or aggregate of molecules of dimensions of several nm (tens of $\AA$ ) or larger. This theme of size matching (or mismatching) recurs often in supramolecular chemistry.

The flavor of supramolecular systems is retained in certain single molecular entities whose atoms are all covalently bonded, but which may be imagined to consist of two (or more) key chemical subunits connected by a flexible or a rigid "spacer". The system may be considered an ordinary molecule if the spacer imbues no special properties beyond those expected from the sum of the properties of the two individual subunits. However, the system becomes supramolecular and the molecule is transformed into a supermolecule when the spacer plays a special role which causes the subunits to behave in a fashion that is unexpected or is qualitatively distinct from the behavior of the subunit in a model molecular environment, such as a non-viscous homogeneous solvent. Let us consider some of these ideas for a pair of radical subunits as shown in Figure 1.

In Figure 1a a radical pair is shown undergoing random diffusional trajectories within the solvent cage of an ordinary molecular, non-viscous solvent. In Figure $1 \mathrm{~b}$ the supramolecular system of a radical pair in a "supercage", such as that provided by a micelle is shown and in Figure 1c the supramolecular system provided by a flexible chain covalently connecting the subunits of a biradical is shown. What makes us consider the situation in Figure 1a "molecular", while we consider the situation in Figures $1 \mathrm{~b}$ and $1 \mathrm{c}$ as supramolecular? This is, of course, an important and generic question. Our approach has been to first employ empirical criteria for answering the question and then after clear cut examples are discovered, they are subjected to theoretical scrutiny and analysis. The empirical criteria are straightforward: after the molecular properties of a system have been defined, any system to be classified as supramolecular must possess either a qualitatively or quantitatively different behavior from the molecular system. In the case of the systems shown in Figure 1, the molecular system possesses some characteristics that are grouped together under the concept of the "cage effect" of liquids. To be a "supercage", i.e., a supramolecular system or a super molecule, some special characteristics must be present. 


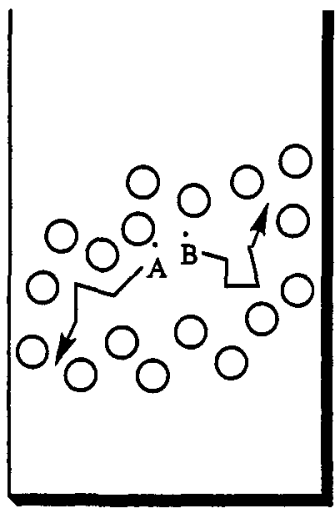

(a) Solvent cage

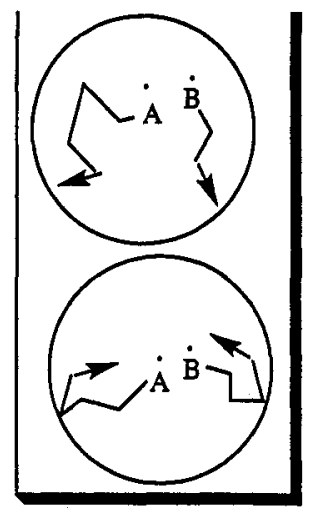

(b) Supercage

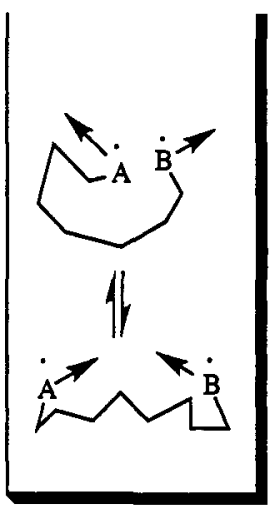

(c) Supermolecule

Figure 1. Comparison of a molecular system (a radical pair in a solvent cage, a) with two supramolecular systems (a radical pair in a supercage, $\mathbf{b}$, and a biradical whose radical fragments are connected by a flexible chain of covalently bonded atoms, c).

\section{Topological Representations of Supramolecular Structures}

Topological representations of chemical systems display only those features which characterize the system at the most fundamental levels of structure, i.e., composition and constitution. ${ }^{2}$ In effect, at the topological level we seek to know the answer to only two questions: (1) what is the composition? and (2) how are the components of the composition connected? Figure 2 shows how chemical structures can be represented topologically convenient in 2 dimensions, an extension to 3 dimensions being straightforward. The typical guest-host supramolecular complex of interest to this report can be represented by a topological object which possesses an inside (I), an outside (O) and a boundary (B). For example, for a single guest, single host system, there are three possible supramolecular isomers (Figure 2 a and c). For two guests and one host there are six topological isomers.

\section{The Cage Effect. From the Solvent Cage to the Supercage.}

We review briefly the development of the "cage effect" in liquids and its extension to the "supercage effect" in micelles as an example of the development of supramolecular concepts and their applications to produce novel and extensive supramolecular photochemical investigations. The "guests" in the cage effect are geminate radical pairs produced by a photochemical event, for example the photochemically induced $\alpha-$-cleavage of triplet ketones. The "host" in the "molecular" cage effect is the restricted space produced by a solvent cage and the "host" in the "supramolecular" cage effect is the restricted space produced by a micellar aggregate.

The paradigm for the $\alpha$-cleavage of ketones is well established and needs no special comment here. ${ }^{3}$ The guest triplet geminate radical pair is produced in high yield, for example, when benzoin and its derivatives are photolyzed. We shall employ methyldesoxy benzoin, a chiral molecule, as an example of supramolecular photochemistry in micellar systems. Before we do that, let us review the history of the development of the classical molecular cage effect of a radical pair produced by a bond dissociation in a liquid.

\section{The Dynamic Radical Pair. The Mechanical Model of Franck, Rabinowitch and Wood.}

When a pair of potentially reactive radicals are formed by the cleavage of a bond connecting the radical partners in an inert liquid, the solvent molecules serve as a "cage" around the geminate (born together) radical pair as discussed by Franck and Rabinowitch ${ }^{4 a}$ and Rabinowitch and Wood.4b The mechanical dynamics of such a system possess some properties which are different in the liquid phase compared to the gas phase. Here are two important quotes from each of these classic papers:

"Compared to the gas phase, the probability of a recombination of a reactive pair of fragments produced by photodissociation is lower in solution because of the action of the solvent cage about the pair. The solvent will remove energy and serve as a hindrance to separation, both of which will enhance the probability of recombination." $4 a$

"Based on a mechanical model of balls in a container, it was concluded that collisions between molecules which are nearest neighbors in a solvent cage occur in sets." $4 b$ 
(a)

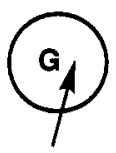

Inside

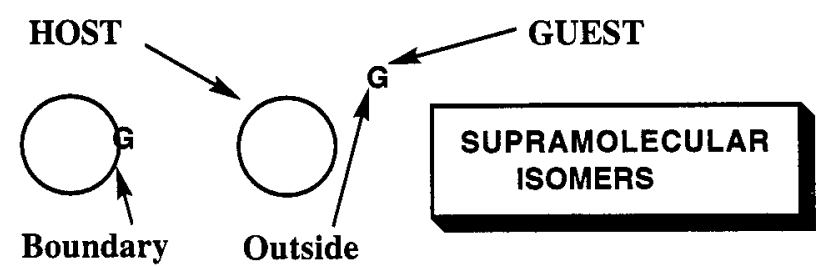

Outside

(b)

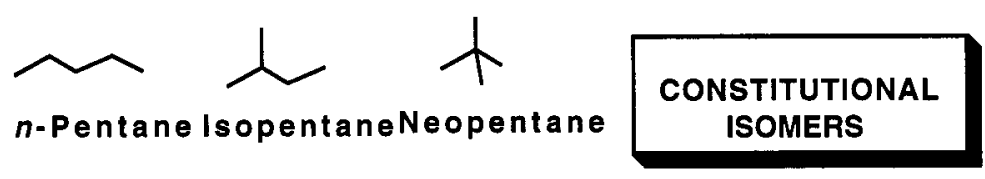

(c) One guest, one host

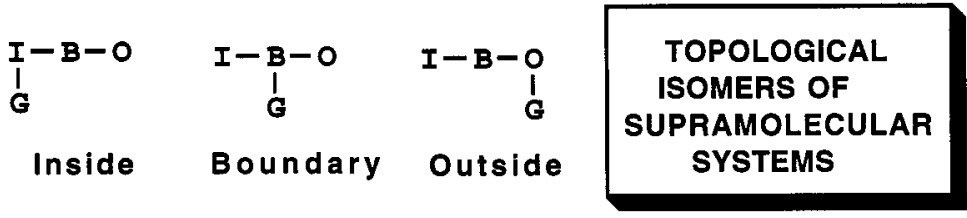

Figure 2. Comparison of the topological representations of molecular structures and supramolecular structures. See text for discussion.

The first, often cited peculiarity of the cage effect has been widely recognized. The latter peculiarities, that collisions occur in sets, were revealed in an ingenious experiment ${ }^{4 \mathrm{~b}}$ consisting of simulating the behavior of molecules in the condensed phase by measuring the behavior of a mechanical device consisting of a series of agitated balls and a knob which was constantly being struck by the balls. The device consisted of a flat brass plate with a zig-zag boundary and a metal knob in the center of the plate which was, however, insulated by being raised slightly from the plate. The knob, made of electrically conducting material, was connected to one pole of a battery, the conducting plate to the other pole of the battery. The circuit could be closed when a metal ball, rolling on the plate struck the knob and thereby removed the insulation of the knob from the plate. One experiment consisted of adding a number of small wooden, non-conducting balls to the plate together with a single metal ball. The conducting knob and the metal ball were intended to simulate two dissolved molecules in a "two dimensional" liquid phase. Every time the metal ball struck the conducting knob, the circuit was closed and current flowed. This allowed the number and frequency of collisions to be measured simply by measuring the number of times the circuit was opened and closed. A lamp was placed in the circuit and its flash signaled a collision! Thus, the "collisions" of molecules were simulated by the collisions of the conducting ball striking the knob in the center of the plate. From this model came the conclusion that collisions between geminate partners occur in sets.

\section{The Reactive Dynamic Geminate Radical Pair. Recombination Reactions.}

When a molecule is dissociated into two reactive radicals (a geminate radical pair) in the liquid phase (as is the case for the $\alpha$-cleavage reaction of many ketones), in the absence of radical scavengers the fragments of the primary geminate pair will eventually undergo "combination" reactions such as recombination and disproportionation. We shall consider only the recombination reaction which regenerates the bond that was initially cleaved. In effect, we are considering a very simple pair of chemical events (Figure 3): (1) the snipping of a carbon-carbon bond resulting from $\alpha$-cleavage and (2) the knitting of the same carboncarbon bond. In order to understand the details of this "snip-knit" process, we must consider some of the features of the reactive, dynamic geminate radical pair ${ }^{5}$ which begins its life in the triplet state immediately after the bond is snipped, yet must undergo intersystem crossing to a singlet geminate radical pair in the state of collision before the bond can be reformed. As shown in Figure 3, the radical pair produced by $\alpha$-cleavage of a triplet ketone possesses some interesting complications, namely two magnetic electron spins and some interacting nuclear spins (e.g., ${ }^{1} \mathrm{H}$ and ${ }^{13} \mathrm{C}$ when this isotope is located at the carbonyl carbon in an enriched sample). ${ }^{6}$ The complications which enter the mechanism because of this triplet to singlet intersystem crossing are simply delicious! We shall see that a remarkably simple and elegant method of examining these complications experimentally is to measure the stereochemistry of the bond that is reformed in the snip-knit sequence, i.e., monitor the racemization of or the diastereoisomerization of appropriate ketones. 


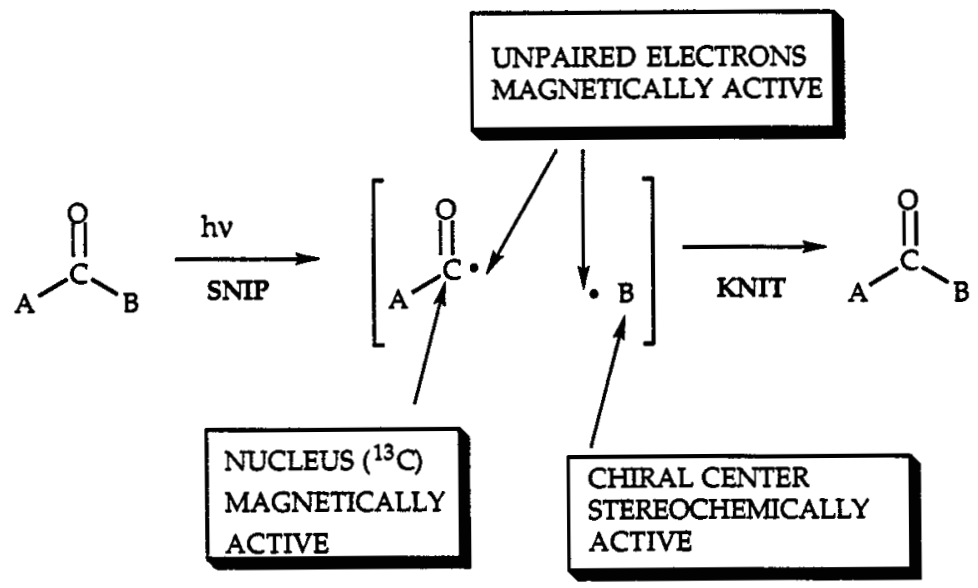

Figure 3. The "snip and knit' strategy for the investigation of the behavior of geminate radical pairs. See text for discussion.

\section{Topological Representations of Two Guest/ One Host Supramolecular Systems: Radical Pairs Adsorbed in Micelles, Donor Acceptor Pairs Adsorbed on Starburst Dendrimers and DNA.}

Figure 4 displays the topological representation of the possible structures of a supramolecular system possessing a composition of two guests $(\mathrm{G})$ and single host (circle). The latter, as in Figure 2, consists of an inside, a boundary and an outside. Ignoring the differences between $G_{1}$ and $G_{2}$ there are 6 possibile "supramolecular constitutional isomers" $\mathbf{a}-\mathbf{f}$. Isomers $\mathbf{a}, \mathbf{b}$ and $\mathbf{c}$ are pertinent to the systems investigated here, i.e., a radical pair adsorbed by a micelle and an electron donor-acceptor pair adsorbed by a starburst dendrimer or by a single strand of DNA. Isomers $\mathbf{d}, \mathbf{e}$ and $\mathbf{f}$ correspond to the separation of one of the guests from the supramolecular structure. Although these structures are interesting and important, for the purpose of this report, the systems discussed correspond mainly to isomers $\mathbf{a}, \mathbf{b}$ and $\mathbf{c}$. We note that structures $\mathbf{a}, \mathbf{b}$ and $\mathbf{c}$ may interconvert during measurement or be distinct during measurement. Each of the structures $\mathbf{a}, \mathbf{b}$ and $\mathbf{c}$ may have conformational isomers, depending on the exact relative locations of each guest molecule within the host or even on the instantaneous shape of the host. We now see that the simple radical pair, in a supramolecular system consists of numerous isomers and even a geminate radical pair must be considered as a minimum of three supramolecular isomers (when the radicals $\mathrm{G}_{1}$ and $\mathrm{G}_{2}$ are different, then two structures correspond to $\mathbf{b}$, i.e., one or the other guests is inside and the other is on the boundary).

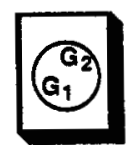

$\Downarrow \mathbf{a}$

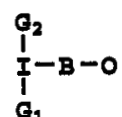
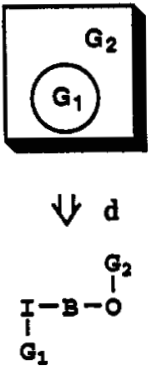
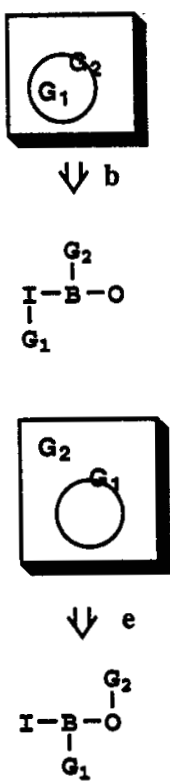
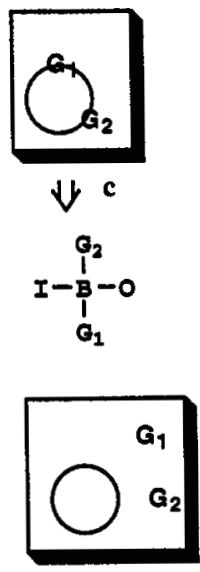

$$
\begin{array}{r}
\forall f \\
I-B-a_{1} \\
G_{2} \\
G_{2}
\end{array}
$$

Figure 4 Topological representations of a supramolecular system whose composition is two guest molecules and one host molecule (or an aggregate of molecules as a host). Structures $\mathbf{a}, \mathbf{b}$ and $\mathbf{c}$ correspond to supramolecularly connected pairs. Examples are geminate radical pairs adsorbed in a micelle, an electron donor-acceptor pair adsorbed on the same starburst dendrimer molecule and an electron donor-acceptor pair adsorbed on the same DNA molecule. 


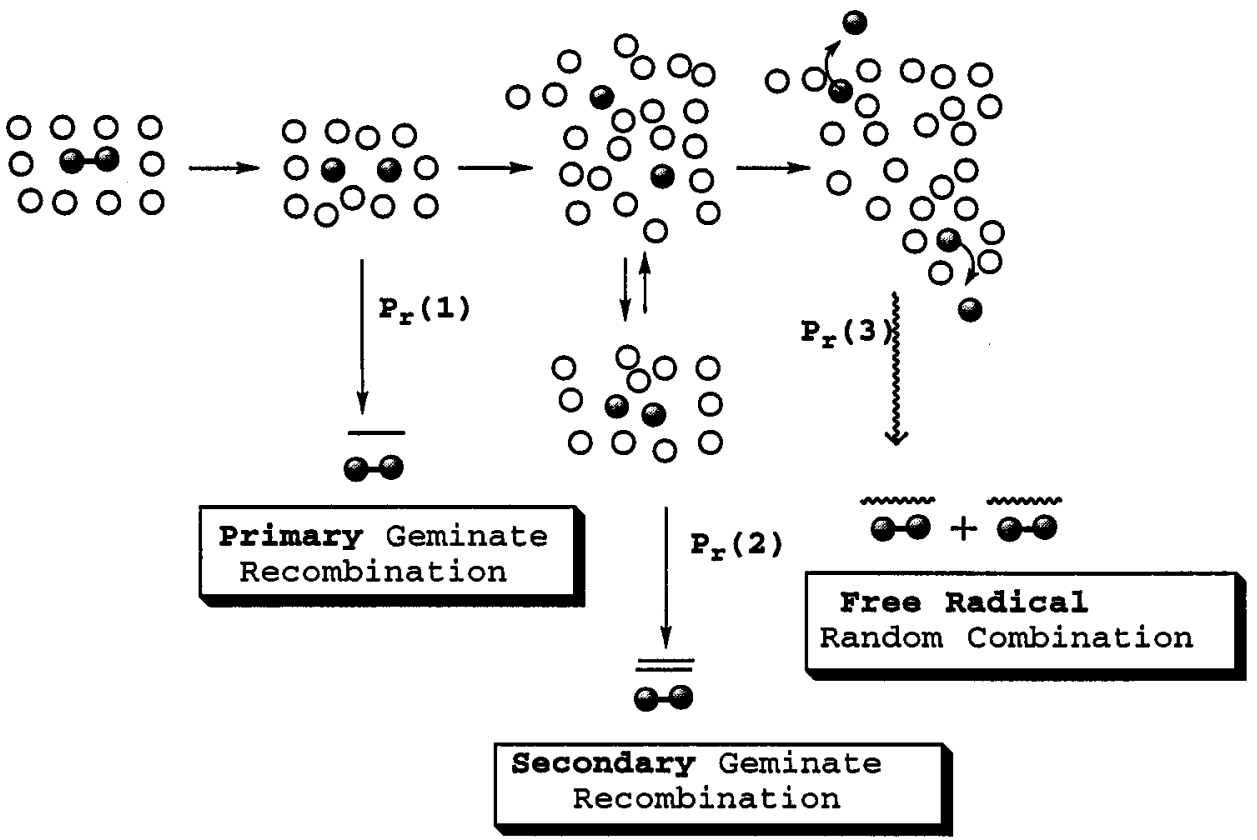

Figure 5. Schematic description of the cage effect. See text for discussion.

To understand how these complications of structure influence the chemistry of a geminate radical pair, and understand how they influence the transition from the solvent cage to the supercage (i.e., from molecular photochemistry to supramolecular photochemistry), we now consider a feature of the dynamic radical pair beyond the sets of collisions classical cage effect proposed by Rabinowitch and Wood. According to the Noyes ${ }^{7}$ random walk model of the dynamic radical pair, the latter, produced by bond dissociation of a molecule, may undergo recombination reaction in one of three conceptually distinct ways as shown in Figure 5: (1) recombination of geminate pairs may occur in the primary cage in which the pair is created with a probability, $\mathrm{P}_{\mathrm{r}}(1)$; (2) recombination of geminate pairs may occur in a secondary cage formed after diffusive excursions with a probability, $\mathrm{P}_{\mathrm{r}}(2)$; or (3) combination of random pairs may occur in secondary cages which form after extensive diffusive excursions with a probability, $\mathrm{P}_{\mathrm{r}}(3)$. In the latter case, combination occurs between random, uncorrelated pairs, not geminate pairs.

The cage effect may be operationally defined as the sum of the primary and secondary geminate recombination reactions, i.e., $P_{r}=P_{r}(1)+P_{r}(2)$. $P_{r} c a n$ be determined experimentally by the measurement of the stereochemistry of a photolyzed ketone as a function of the extent of reaction and how the experimental value of $\mathrm{P}_{\mathrm{r}}$ determined in this manner can be related to a theoretical value which is computed based on a model for the dynamic radical pair.

\section{Experimental Determination of $\mathbf{P}_{\mathbf{r}}$}

Buchachenko and Tarasov showed that the value of $\mathrm{P}_{\mathrm{r}}$ may be determined experimentally 8 by measuring a recombination efficiency parameter, $\beta$, where $\beta=\left[\mathrm{P}_{\mathrm{r}} /\left(1-\mathrm{P}_{\mathrm{r}}\right)\right] . \quad \beta$ is by determining the loss of stereochemistry of a chiral ketone (Figure 6 , methyldesoxybenzoin) or a diastereomer (Figure 7 ,

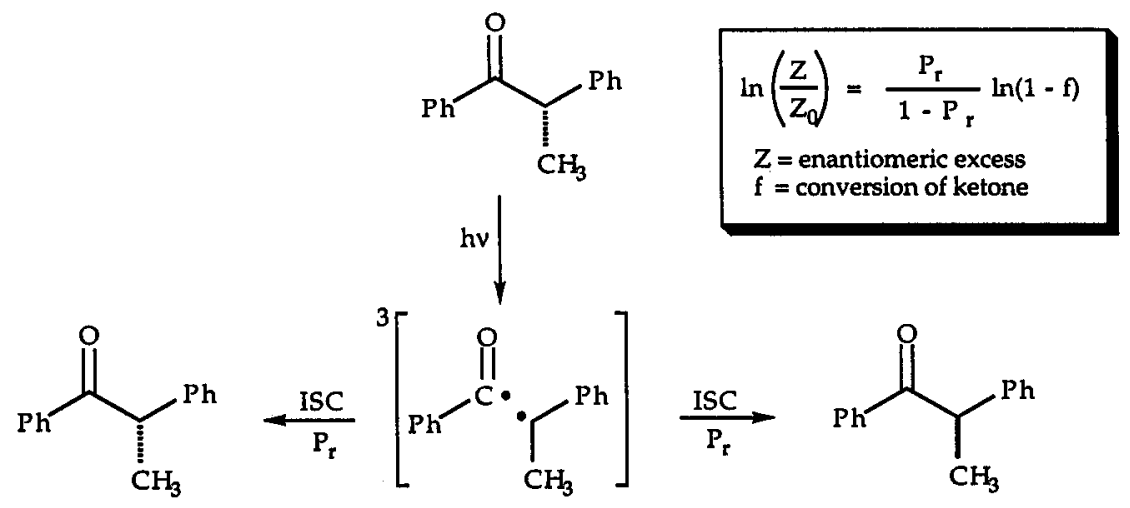

Figure 6. Recombination of the geminate radical pair produced by the photolysis of methyldesoxybenzoin (MDB). Other combination and disproportionation products are formed in minor yields and are ignored. 


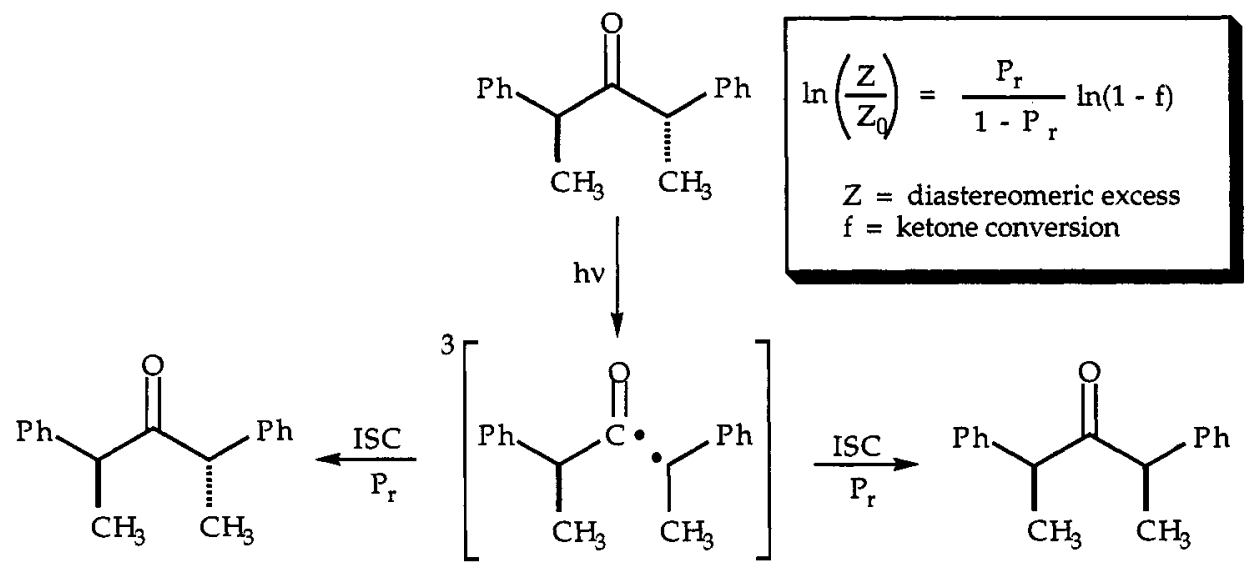

Figure 7. Recombination of the geminate radical pair produced by the photolysis of 2,4-diphenylpentanone (DPP). Other combination and disproportionation products are formed in minor yields and are ignored.

diphenylpentanone). The magnitude of $\beta$ is derived from an appropriate plot (see inserts of Figures 6 and 7). From the relationship of $\beta$ to $P_{r}$, the experimental value of $P_{\mathbf{r}}$ is evaluated.

\section{Theoretical Determination of $\mathbf{P}_{\mathbf{r}}$}

The value of $\mathrm{P}_{\mathrm{r}}$ may be determined theoretically by computing the recombination probability from a model of a dynamic radical pair. ${ }^{9}$ The model must include explicit forms for the magnetic spin dynamics (triplet to singlet intersystem crossing), the details of the random walk of the radical pair (modulation of the exchange interaction as a function of separation) and the chemical dynamics (only collisions of singlet pairs lead to recombination). The key magnetic parameters incorporated into the spin dynamics of the model are the magnitude of an applied external magnetic field (if any), the hyperfine couplings of the magnetic nuclei embedded in the pair, the g-factors of the individual radicals of the pair and other magnetic interactions (e.g., spin-orbit coupling) that might lead to intersystem crossing of the triplet pair.

In addition to these magnetic parameters, the intersystem crossing efficiency is also influenced by other factors such as the rates of reencounters and the modulating, distance dependent exchange interaction which tends to "quench" intersystem crossing when the pair is in the state of collision. Finally, the intersystem crossing is strongly influenced by the size and "viscosity" of the supercage in which the pair is embedded. In the case of a micelle, a specific model which includes these factors and others, such as the probability of one of the pairs leaving the micelle when it approaches the micellar boundary, have been developed.

A qualitative feel for the model for the radical pair in a micelle can be obtained by consideration of Figure 8. The micelle is modeled as a liquid sphere with a moderate viscosity. 9,10 The diffusional dynamics of the radical pair is simulated by maintaining one of the partners in the center of the micelle and allowing the other to execute a random walk in the micellar space. When the random walking partner approaches the micellar boundary there is certain probability it will leave the micelle and pop into the aqueous phase. The concept of snip and knit are correlated with the diffusional trajectory of the walker and is shown in Figure 8 around the periphery of the sphere. The micellar size, micellar viscosity, the time scale of intersystem crossing and time of duration of the walk are critical in determining the value of $\mathrm{P}_{\mathrm{r}}$, as outlined in Figure 8 (see the original papers for details).

\section{Comparison of the Experimental and Theoretical Values of $\mathrm{Pr}$ as a Function of Micelle Size}

The experimental values of $P_{r}$ were determined ${ }^{9,10}$ for the methyldesoxybenzoin(MDB) and 2,5diphenylpropanone (DPP) systems as a function of micelle size for micelles consisting of anionic surfactants containing $8,9,10,11$ and 12 catrbon atoms in the surfactant chain. The rather startling observation of the results is that for MDB the value of $P_{r}$ increases as the size of the micelle increases, whereas for DPP the value of $P_{r}$ decreases as the size of the micelle increases. Such a qualitatively different behavior provides a real challenge to the theory. However, a reasonable agreement has been achieved for each of these systems. 9,10 


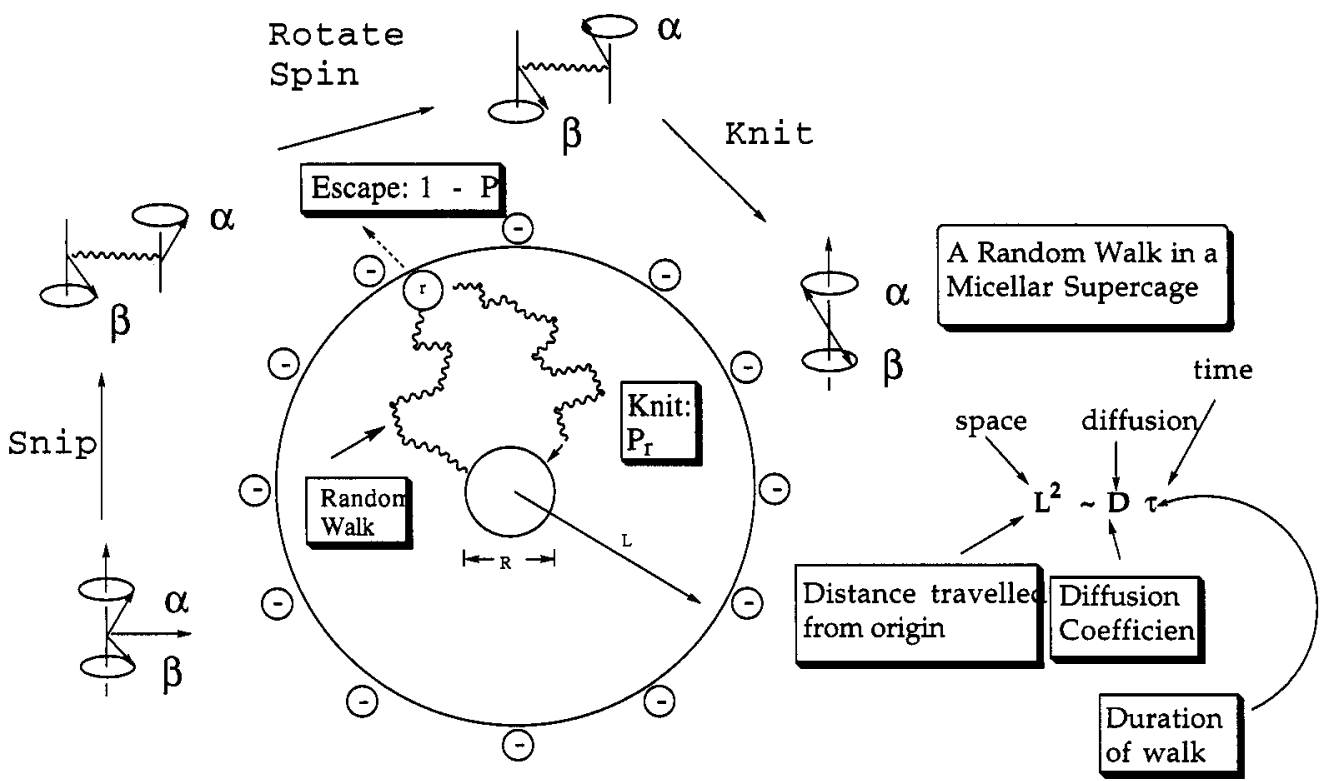

Figure 8. Schematic representation of the model employed to compute the value of $\mathrm{P}_{\mathrm{r}}$ for the recombination (snip-knit) reaction of a geminate radical pair in a micelle. See text for discussion.

\section{Paradigm and Parameters for Photoinduced Electron Transfer Between Metal Complexes Bound to Anionic Interfaces}

We have employed photoinduced electron transfer to investigate the structure and dynamics of supramolecular systems composed of metal complexes (guests) and anionic interfaces (hosts). ${ }^{11}$ The structures of the metal complexes which served as donor and acceptor are familiar to photochemists as they are derivatives related to the famous $\mathrm{M}(\mathrm{bpy}) 3^{+\mathrm{n}}$ structures. As a typical donor $\mathrm{M}=\mathrm{Ru}, \mathrm{n}=2$; and as a typical acceptor $M=R h, n=3$.

The photoinduced transfer between donor-acceptor pairs of such metal complexes bound to three different anionic host interfaces provided by micelles, starburst dendrimers, and (synthetic and natural) DNA were investigated. From these studies we have determined the binding modes of the guest-host complexes and the rate constants for electron transfer as a function of binding mode and the size and dimensionality of the host. A brief survey of the results for photoinduced electron transfer of the donor-acceptor pairs adsorbed on starburst dendrimers and DNA will be presented here. The referenced literature will allow the interested reader to probe the results further.

\section{Starburst Dendrimers}

Starburst dendrimers are supermolecules with precise compositional and constitutional structures of nanoscopic dimensions (Figure 9). ${ }^{12}$ The size of the smallest dendrimers employed were of the order of 3 $\mathrm{nm}$ in diameter ( $30 \AA)$ and the largest were of the order of $20 \mathrm{~nm}(200 \AA)$. The central vertex of starburst dendrimer is a nitrogen atom from which radiates branches resulting from sequential addition-condensation reactions. The outside core consists of carboxylate groups, so that the external surface is negatively charged, just like an SDS micelle.

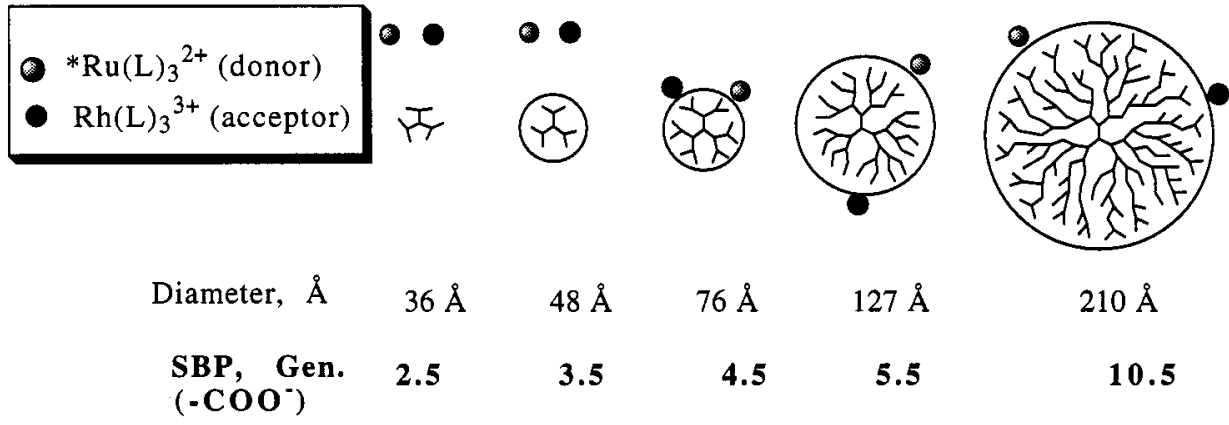

Figure 9. Schematic representation of the size of the donor-acceptor (guests) metal complexes and several generations of starburst dendrimers (host). 
In collaboration with Professor Donald Tomalia of the Michigan Molecular Institute, we have found that the photoinduced electron transfer of donor-acceptor complexes adsorbed on the ionic surface of starburst dendrimers 13 indicates that the electron transfer occurs nearly exclusively between pairs which are located on the same dendrimer molecule after the dendrimer reaches a certain critical size. In these cases the rate constants extracted from the data were cleanly first order. In some respects, the electron transfer process is similar to the "cage" reaction of radical pairs in micelles. A detailed analysis of the data showed that the rate constant of the electron transfer process decreased as the dendrimer size increased. Two possibilities were considered as explanations of these results: (1) with increasing dendrimer size the surface increases, effectively "diluting" the concentration of the acceptor and thereby decreasing the magnitude of the measured unimolecular rate constant; (2) with increasing dendrimer size the surface "viscosity" increases, the quenching reaction is diffusion controlled, and the decreasing rates are due to decreasing rates of diffusion.

Investigation of the Photoinduced Electron Transfer in $D_{x}-[D N A]_{n}-A y$ Supermolecules In collaboration with Professor Jacqueline K. Barton of Caltech, we have investigated ${ }^{14}$ photoinduced electron transfer between metal complexes that are non-covalently bound to synthetic and natural DNAs. We have found that the rate constants for electron transfer are strongly influenced by the ligand structure of complexes which exhibit only small (bimolecular) rate constant differences in homogeneous solutions. We have interpreted these results to be due to different binding modes of the guest-host supramolecular system (Figure 10): the donor and acceptor complexes may bind to ionic (atmospheric) sites near the aqueous/DNA interface, to surface sites near the hydrophobic base pairs and to intercalating sites between the base pairs. The transport of the complexes along the DNA double helix is expected to be a strong function of the mode of guest binding and, therefore, to strongly influence the rate constant of energy transfer if it is transport dependent.

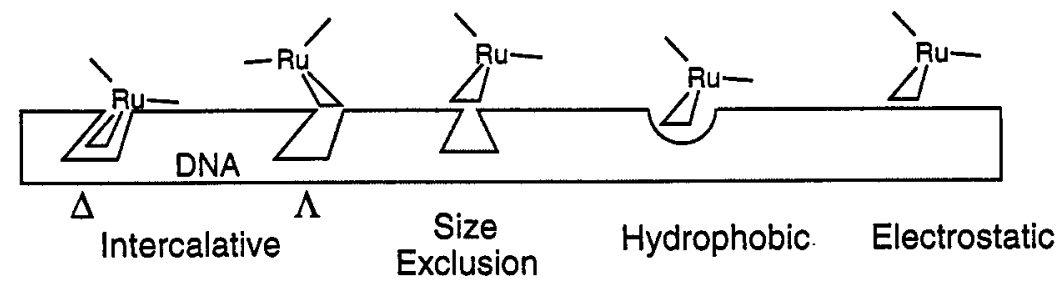

Figure 10. Schematic description of various binding modes of metal complexes to DNA. See text for discussion.

We wondered whether we could achieve evidence for the interesting possibility that electron transfer may occur over relatively long distances between two spacially separated donor-acceptor pairs if the DNA host can serve as an "electron conducting wire". However, all of our attempts to demonstrate long range transfers were hampered by the ambiguity resulting from the lack of precise information on distance relationships between complexes which are non-covalently associated with the DNA. For example, an unusually fast rate of electron transfer compared to that observed in homogeneous solution is an earmark of a supramolecular system, but the mechanism of the enhancement could be preorganization of the donor acceptor pairs, reduction of the transport dimensionality due to bonding to DNA or the more exciting coupling of the donor and acceptor by the DNA double helix.

Therefore, we decided to attempt to overcome the ambiguity of distance relationships by synthesizing DNA structures to which the donor and acceptor are covalently bound to the DNA in a precisely determinable manner from which the distance between donor and acceptor could confidently be estimated. The experimental strategy was to synthesize a tris-polypyridyl ("linking") ligand which is functionalized so that it can be attached to the 3' position of a single strand of synthetic DNA. Donor and acceptor complexes containing this ligand and ancillary ligands were synthesized, and the donor and acceptor were each linked to the $3^{\prime}$ end of a single stranded DNA. The strategy was that two labeled single strands will then hybridize thereby producing a DNA bridged donoracceptor supermolecule $\mathbf{D}_{\mathbf{x}}$-[DNA $]_{\mathbf{n}}-\mathbf{A y}$ (where $n$ is the number of base pairs separating the point of covalent attachment of $D$ and $A, x$ is the number of atoms in a flexible tether connnecting $D$ to its DNA strand, and $y$ is the number of atoms in a flexible tether connecting $A$ to its strand). The composition and nature of the DNA bridge and the distance between donor and systematically through modern DNA oligomer synthetic techniques. Important systematic investigations based on this strategy would include variation of the number and chemical structure of base pairs for a given donor and acceptor pair and variation of the acceptor metal center for a given DNA bridge.

Synthesis of such a matrix of D-[DNA $]_{n}-A$ molecules is a very ambitious and potentially difficult synthetic program. However, we have demonstrated ${ }^{15}$ the feasibility of the strategy by synthesizing a metal complex (Figure 11), possessing a linking ligand which will covalently attach the complexes to the strands of a 15 mer DNA (the size of which is known to be comparable to that of the major groove of natural 


\section{ELECTRON TRANSFER THROUGH DNA}

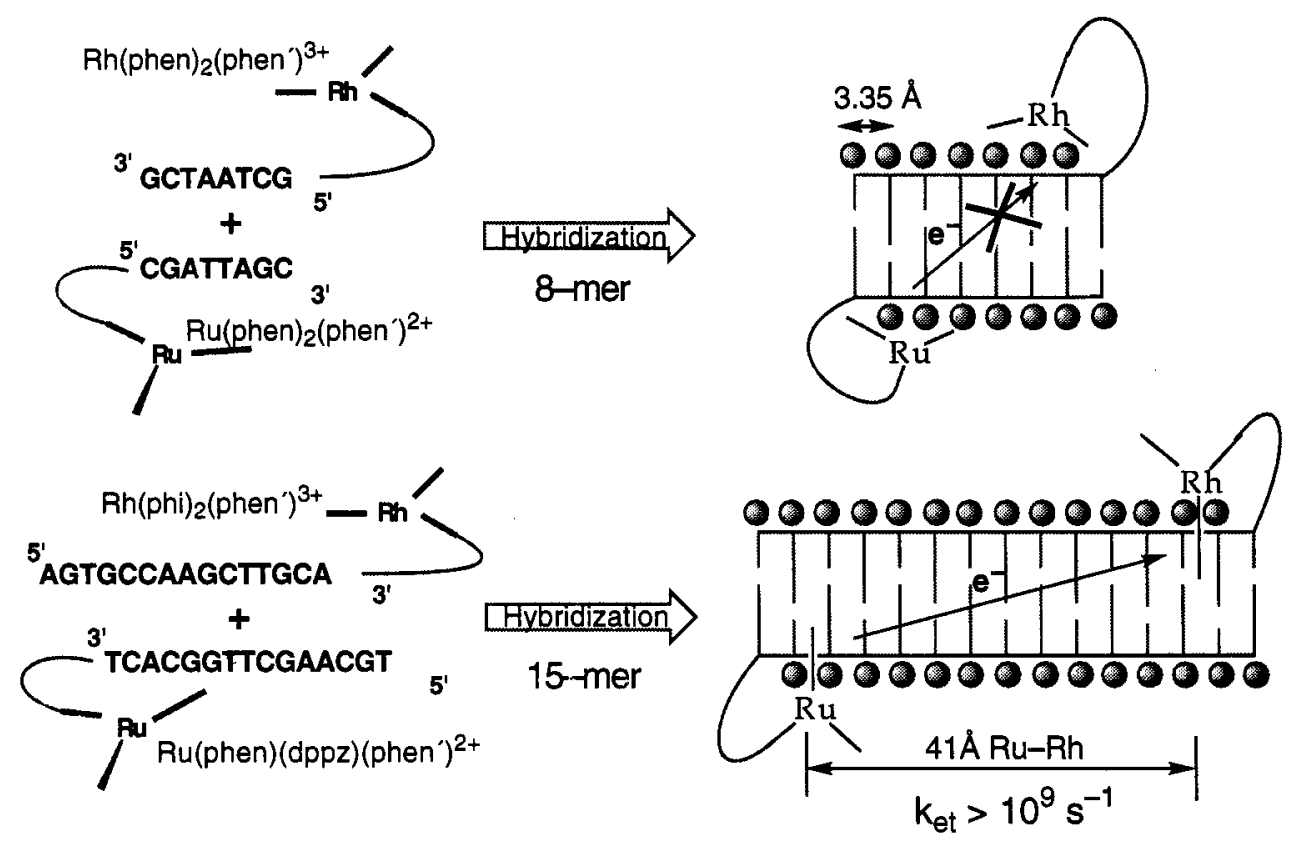

Figure 11. Schematic description of the "short" and "long" DNA supermolecules investigated. See text for discussion.

DNA). One of the ligands coordinated to both the donor and the acceptor were selected from those ligands known to be excellent intercalators of DNA. From molecular models it is possible that both the donor $\mathrm{Ru}$ moiety and the acceptor Rh moiety can intercalate between base pairs of the 15 mer (shown schematically in Figure 11). If the intercalation actually occurs, the supermolecule was expected to be an excellent probe for the investigation of the DNA spacer as a"wire" for the photoinduced electron transfer between D and $\mathrm{A}$, since the spacing between the metal centers is of the order of $30 \AA$ for such a structure. Indeed, nanosecond laser flash spectroscopy reveals that the rate of quenching of the excited donor is remarkably fast (ca. $10^{9} \mathrm{~s}^{-1}$ !). This requires an extraordinary rate of electron transfer for a donor and acceptor separated by such a distance. Ordinary electron transfer theory predicts a rate that is orders of magnitude smaller.

It was suspected that the intercalation of a ligand into the DNA double helix might be a requirement for the DNA host to behave as a "wire" for the electron transfer. To test for this possibility, a smaller DNA (an 8 mer) was synthesized, the donor and acceptor were again covalently attached to the opposite ends of the double helix, but this time each complex possessed only non-intercalating ligands. Indeed, although the donor and acceptor were much closer (ca. $15 \AA$ ) in the 8 mer, there was no detectable quenching of the Ru excited state in the supermolecule! This is a rather convincing demonstration of the requirement of intercalation and the involvement of the DNA as a conducting wire in the electron transfer event.

\section{Summary}

Supramolecular photochemistry offers the photochemist an opportunity to both quantitatively and qualitatively modify the photochemistry of systems whose molecular photochemistry has been well established. Thus, a famous secondary photochemical process, the geminate combination reactions of radical pairs and a famous primary photochemical process, the electron transfer between a photoexcited donor and a ground state acceptor, could be used to probe deeply the structure of micelles on the one hand and the structure of starburst dendrimers and DNA on the other. The notion that true supramolecular systems require more than an understanding of the additive chemistry of the molecular systems is clearly evident in these investigations. In the case of radical pairs in supercages, the role of interacting spin, molecular and chemical dynamics and the size of the supercage are made clear by a quantitative model.

Acknowledgment. The author is grateful to the previous Porter Medalists for serving as inspiring role models for him during his graduate research studies and the launching of his own independent career in photochemistry. He remembers searching the literature avidly for the next paper coming from the laboratories of Porter, Weller, or Kasha, because of the valuable insights that these papers contained and because of the exciting new areas that each explored. Our community is blessed to have such outstanding scientists and intellects among us. 
The author thanks the many students, postdocs, visitors, and collaborating colleagues which whom he has had the privilege to work with and learn from. The senior colleagues involved in these collaborations described in this report, Professors Barton, Buchachenko, Tarasov and Tomalia, deserve special thanks for their friendship, enthusiasm for science, and for allowing him to extend the breadth of his intellectual excitement to horizons that would only have been pleasant but unrealizable scientific fantasies without their involvement.

The National Science Foundation and the Air Force Office of Scientific Research have been generous and faithful supporters of photochemical research at Columbia.

\section{References}

1. (a) F. Vogtle, Supramolecular Chemistry; Wiley: New York, 1991; (b) J.-L. Lehn, Angew. Chem. Int. Ed. Eng. 27, 123 (1988); (c) Frontiers in Supramolecular Organic Chemistry and Photochemistry; H. J. Schneider, H. Dürr, Eds.; VCH: Weinheim, Germany, 1991; (d) V. Balzani, F. Scandola,Supramolecular Photochemistry; Prentice-Hall: New York, 1991.

2. (a) N.J. Turro, Angew. Chem. Int. Ed., Engl., 25, 882 (1986); (b) N.J. Turro and M. Garcia-Garibay, in Photochemistry in Organized and Constrained Media, ed. V. Ramamurthy, VCH Publishers, 1991.

3. N. J. Turro, Modern Molecular Photochemistry; University Science Books: Mill Valley, CA, 1991.

4. (a) J. Franck and E. Rabinowitch, Trans. Faraday Soc., 30, 120 (1934); (b) E. Rabinowitch and W. C. Wood, Ibid., 32, 1381 (1936).

5. For an excellent discussion of the dynamic geminate radical pair see K. M. Salikov, Y. N. Molin, R. Z. Sagdeev, and A. L. Buchachenko. Spin Polarization and Magnetic Effects in Radical Reactions. Amsterdam: Elsevier (1984).

6. Our research group began its investigation of radical pairs and the cage effect in micelles in the late 1970s. For reviews see: (a) N.J. Turro and B. Kraeutler, Acc. Chem. Res., 13, 369 (1980); (b) N.J. Turro, Proc. Natl. Acad. Sci. USA, 80, 609 (1983); (c) N.J. Turro and B. Kraeutler, "Magnetic Isotope Effects," in Isotopic Effects, vol. 6, E. Buncel and C.C. Lee, Eds., Elsevier, Amsterdam, 1984, pgs. 107-160; (d) I.R. Gould, N.J. Turro and M.B. Zimmt, Adv. Phys. Org. Chem., 20, 1 (1984).

7. R. M. Noyes, J. Am. Chem. Soc. 77, 2042 (1955).

8. V. F. Tarasov, and A. L. Buchachenko, Bull. Acad. Sci., USSR, 36, (Part 1), 500 (1987).

9. V.F. Tarasov, N.D. Ghatlia, A.L. Buchachenko and N.J. Turro, "J. Am. Chem. Soc., 114, 9517 (1992).

10. V.F. Tarasov, N.D. Ghatlia, N.I. Avdievich, I.A. Shkrob, A.L. Buchachenko and N.J. Turro, J. Am. Chem. Soc., 116, 2281 (1994).

11. N. J. Turro, J. K. Barton and D. A. Tomalia, Acc. Chem. Res., 24, 332 (1991).

12. D. Tomalia, Angew. Chem. int. ed. Eng., 29, 138(1990).

13. (a) M. Moreno-Bondi, G. Orellana, N.J. Turro and D.A. Tomalia, Macromolecules, 23, 910 (1990); (b) K.R. Gopidas, A.R. Leheny, G. Caminati, N.J. Turro and D.A. Tomalia, J. Am. Chem. Soc., 113, 7335, 1991.

14. (a) J.K.Barton, C.V. Kumar, and N.J. Turro, J. Am. Chem. Soc., l08, 6391 (1986);

(b) M.D. Purugganan, C.V. Kumar, N.J. Turro and J.K. Barton, Science, 241, 1645 (1988);

(c) A.M. Pyle, J.P. Rehmann, R. Meshoyrer, C.V. Kumar, N.J. Turro and J.K. Barton, J. Am. Chem. Soc., 111, 3051, (1989).

15. C.J. Murphy, M.R. Arkin, Y. Jenkins, N.D. Ghatlia, S.H. Bossmann, N.J. Turro and J.K. Barton, Science, 262, 1025 (1993). 